\title{
MATRIX PRODUCTS OF MATRIX POWERS
}

\author{
R. F. CLIPPINGER
}

1. Introduction. Let $m n$-by- $n$ matrices, $A_{k}$, of complex constants, $a_{i j k}(i, j=1,2, \cdots, n ; k=1,2, \cdots, m)$, be given. We shall denote by $\mathcal{L}$ the set of all matrices,

$$
A(t)=\sum_{i=1}^{m} \rho_{i}(t) A_{i},
$$

where $\rho_{i}(t)(i=1,2, \cdots, m)$ are arbitrary, non-negative, summable functions of the real variable $t$ on the interval $T, a \leqq t \leqq b$. We shall call $\mathfrak{J}, \mathcal{S}$, or $\mathcal{X}$ the subsets of $\mathcal{L}$ obtained by restricting the functions $\rho_{i}(t)$ to polynomial functions, step functions, or step functions which are all zero except one. Since, in each case, the elements of $A(t)$ are summable functions of $t$ on $T$, it follows that, on $T$, there exists a unique, absolutely continuous matrix solution, ${ }^{1} Y(t)$, of the linear, matrix differential equation and initial condition:

$$
d Y(t) / d t=Y(t) A(t), \quad Y(a)=E,
$$

where $E$ is the $n$-by- $n$ unit matrix. We shall denote by $\lambda, \iota, \sigma$ or $\xi$ the set of matrices, $Y(t)$, which are particular values of solutions of (1.1), where $A(t)$ is an arbitrary matrix of $\mathcal{L}, \mathfrak{J}, \mathcal{S}$, or $\mathcal{X}$, respectively, and $t$ is on $T$.

If $A$ is a matrix with elements $a_{i j}$, let the absolute value of $A$ and the exponential and natural logarithm of $A$ be defined ${ }^{2}$ by the equations:

$$
\begin{aligned}
|A| & =\left[\sum_{i, j=1}^{n}\left|a_{i j}\right|^{2}\right]^{1 / 2}, \\
\exp A & =\sum_{i=0}^{\infty} A^{i} / i ! \\
\log A & =\sum_{i=1}^{\infty}(-1)^{i-1}(A-E)^{i / i}, \quad \text { if } \quad|A-E|<1 .
\end{aligned}
$$

Presented to the Society, April 18, 1942; received by the editors April 4, 1943, and, in revised form, August 18, 1943. The author wishes to thank Professor G. D. Birkhoff for suggestions which led to this paper.

1 See W. M. Whyburn, On the fundamental existence theorems for differential systems. Ann. of Math. (2) vol. 30 (1928-29) p. 31. We observe that equations (1.1) are equivalent to a system of $2 n$ real, linear, first order differential equations satisfying all the hypotheses of this theorem.

2 See J. v. Neumann, Über die analytischen Eigenschaften von Gruppen linearer Transformationen und ihrer Darstellungen. Math. Zeit. vol. 30 (1929) pp. 6, 7. 
J. v. Neumann ${ }^{8}$ has shown that if $j$ is an integer and if $|A-E|<1$, then

$$
A^{j}=\exp [j \log A]
$$

Hence, for any $\alpha$, we define the $\alpha$ power function of $A$ by the equation

$$
A^{\alpha}=\exp (\alpha \log A), \quad \text { if }|A-E|<1 .
$$

If $\left|\exp A_{i}-E\right|<1(i=1,2, \cdots, m)$, we define $\mu$ as the set of matrix products of matrix powers,

$$
\prod_{j=1}^{J} \prod_{i=1}^{m}\left(\exp A_{i}\right)^{\alpha_{i j}}
$$

where the $\alpha_{i j}$ are arbitrary non-negative numbers.

Let us identify an $n$-by- $n$ matrix, $B$, of complex numbers with the point in $2 n^{2}$-Euclidean space, whose coordinates are the real and imaginary parts of the elements of $B$. The distance between two points $B_{1}$ and $B_{2}$ may be defined as $\left|B_{1}-B_{2}\right|$. A set, $\beta$, of matrices, $B$, is then also a point set whose closure we denote by $\bar{\beta}$.

It is the purpose of this paper to show that the sets $\bar{\lambda}, \bar{i}, \bar{\sigma}, \bar{\xi}$ and, if it exists, $\bar{\mu}$, are identical.

\section{Principal theorems.}

THEOREM 2.1. The sets $\bar{\lambda}$ and $\bar{\sigma}$, defined above, are identical.

Since any step function on $T$ is summable on $T$, it follows that $\sigma \subset \lambda$. Suppose $A_{\mathrm{L}}(t)$ is a matrix of class $\mathcal{L}$ with coefficients $\rho_{i}(t)$. Then, for all positive $\delta$, there exists a matrix, $A_{\mathrm{s}}$, of class $\delta$ with coefficients $r_{i}(t)$ such that

$$
\sum_{k=1}^{m} \int_{a}^{b}\left|\rho_{k}(t)-r_{k}(t)\right|\left|a_{i j k}\right| d t<\delta \quad(i, j=1,2, \cdots, n) .
$$

Let the corresponding solutions of (1.1) be $Y_{\mathrm{L}}(t)$ and $Y_{\mathrm{B}}(t)$. Since $Y(t)$ is a uniformly continuous functional ${ }^{4}$ of $A(t)$, it follows that, given any positive number $\epsilon, \delta$ may be so chosen that

$$
Y_{\mathbf{L}}(t)-Y_{\mathrm{S}}(t) \ll \epsilon ;
$$

that is, the absolute value of each element of the matrix on the left is less than $\epsilon$. Hence $\lambda \subset \bar{\sigma}$.

${ }^{3}$ Loc. cit. pp. 8, 12.

${ }^{4}$ By this we mean that the elements of $Y(t)$ are uniformly continuous functionals of the elements of $A(t)$. See W. M. Whyburn, Functional properties of the solutions of differential systems. Trans. Amer. Math. Soc. vol. 32 (1930) p. 508. 
THEOREM 2.2. The sets $\bar{\sigma}$ and $\bar{\xi}$ are identical.

Clearly $\xi \subset \sigma$.

J. v. Neumann ${ }^{5}$ has shown that if $|A|,|B|<(1 / 2) \log (3 / 2)$, then $\log [\exp A \exp B]=A+B+O(|A||B|)$.

This equation, by induction, leads to the generalized equation:

$$
\log \prod_{i=1}^{m} \exp A_{i}=\sum_{i=1}^{m} A_{i}+\sum_{i, j=1}^{m} O\left(\left|A_{i}\right|\left|A_{j}\right|\right),
$$

if $\left|A_{i}\right|<\delta(m)$, where it suffices to take $\delta(m)<[\log (3 / 2)] / 2 n(m-1)$. To this we may add the equation,

$$
\exp (A+B)=\exp A+O(|B|)
$$

which follows immediately from the definition of $\exp (A+B)$.

LEMMA 2.1. If $A$ is a matrix of constants, the matrix,

$$
Y(t)=Y_{0} \exp [(t-a) A],
$$

is the solution of the linear, matrix differential equation and initial condition

$$
d Y(t) / d t=Y(t) A, \quad Y(a)=Y_{0} .
$$

The series $\sum_{j=0}^{\infty}(t-a)^{i} A^{i} / j$ is uniformly convergent $t^{6}$ on any interval $|t-a|<N$, hence the lemma may be established by term-by-term differentiation.

LEMMA 2.2. If $A(t)$ is a matrix of summable functions, and if $A(t) \ll(M)$ on $T$, the solution, $Y(t)$, of equation (1.1) satisfies the inequality

$$
Y(t)-E \ll(1 / n[\exp M n(t-a)-1]) \text { on } T \text {. }
$$

Slight modifications of the proof of the existence theorem given by G. D. Birkhoff and R. E. Langer ${ }^{7}$ yield this lemma.

LEMMA 2.3. If $B_{1}, B_{2}, \cdots, B_{m}$ are square matrices,

$$
\lim _{j \rightarrow \infty}\left[\prod_{i=1}^{m} \exp \left(B_{i} / j\right)\right]^{j}=\exp \sum_{i=1}^{m} B_{i}
$$

${ }^{5}$ Loc. cit. pp. 13-15.

- See J. v. Neumann, loc. cit. p. 7.

${ }^{7}$ The boundary problems and developments associated with a system of ordinary linear differential equations of the first order. Proceedings of the American Academy of Arts and Sciences vol. 58 (1922-1923) pp. 59-63. 
Let $P_{j}=\prod_{i=1}^{m} \exp \left(B_{i} / j\right)$. By Lemma $2.1, P_{j}$ is the solution for $t=1$ of the differential equation

$$
d Y(t) / d t=Y(t) \sum_{i=1}^{m} \rho_{i}(t) B_{i}, \quad Y(0)=E,
$$

where the $\rho_{i}(t)$ are all zero except on the interval $(i-1) / m \leqq t \leqq i / m$ where $\rho_{i}(t)=m / j(i=1,2, \cdots, m)$. Let $B$ be an upper bound to the absolute values of the elements of $m B_{i}$. Then $\sum_{i=1}^{m} \rho_{i}(t) B_{i} \ll(B / j)$. By Lemma 2.2, $j$ may be chosen so large that $\left|P_{j}-E\right|<1$ and $\left|B_{i} / j\right|<[\log (3 / 2)] / 2 n(m-1)$. Hence by $(1.2)$

$$
P_{i}=\exp \left[j \log \prod_{i=1}^{m} \exp \left(B_{i} / j\right)\right] .
$$

From (2.1) it follows that

$$
j \log \prod_{i=1}^{m} \exp \left(B_{i} / j\right)=\sum_{i=1}^{m} B_{i}+O(1 / j) ;
$$

and, finally, (2.2) implies that

$$
P_{i}=\exp \sum_{i=1}^{m} B_{i}+O(1 / j) .
$$

This establishes Lemma 2.3.

Lemma 2.4. If each of $K n$-by-n matrices, $C_{k}$, is the limit of a sequence, $\left\{C_{k j}\right\}$, of matrices, then

$$
\lim _{\jmath \rightarrow \infty} \prod_{k=1}^{K} C_{k j}=\prod_{k=1}^{K} C_{k}
$$

This lemma may be established easily by induction.

To prove that $\sigma \subset \bar{\xi}$, let $Y_{\mathrm{s}}$ be a matrix of $\sigma$; then $Y_{\mathrm{s}}$ is a product of a finite number of matrices of the form

$$
C_{k}=\exp \sum_{i=1}^{m} \alpha_{i k} A_{i}
$$

and by Lemma 2.3 , each $C_{k}$ is a limit of matrices

$$
C_{k j}=\left[\prod_{i=1}^{m} \exp \left(\alpha_{i k} A_{i} / j\right)\right]^{j} .
$$

Hence, by Lemma 2.4, $Y_{\mathrm{S}}$ is a limit of matrices of $\xi$ and therefore $Y_{\mathrm{S}}$ is a member of $\bar{\xi}$. 
Corollary to Theorem 2.2. If $\left|\exp A_{i}-E\right|<1(i=1,2, \cdots ; m)$, then $\xi=\mu$.

To prove this, we need only observe that, by definition,

$$
\left(\exp A_{i}\right)^{\alpha_{i k} / i}=\exp \left(\alpha_{i k} A_{i} / j\right) \quad \text { if } \quad\left|\exp A_{i}-E\right|<1 .
$$

Theorem 2.3. The sets $\bar{\lambda}$ and $\bar{\imath}$ are identical.

Since $\Im \subset \mathcal{L}, \iota \subset \lambda$. Given a matrix in $\mathcal{L}$ with coefficients $\rho_{i}(t)$, there exists a matrix in $\mathfrak{J}$, with coefficients $\mu_{i}(t)$, such that

$$
\sum_{k=1}^{m} \int_{a}^{b}\left|\rho_{k}(t)-\mu_{k}(t)\right|\left|a_{i j k}\right| d t
$$

is arbitrarily small $(i, j=1,2, \cdots, n)$. Therefore ${ }^{8} \iota \subset \bar{\lambda}$.

Carnegie Institute of Technology

${ }^{8}$ See W. M. Whyburn, Functional properties of the solutions of differential systems Loc. cit. 